\title{
Efficacy of follitropin-alpha versus human menopausal gonadotropin for male patients with congenital hypogonadotropic hypogonadism
}

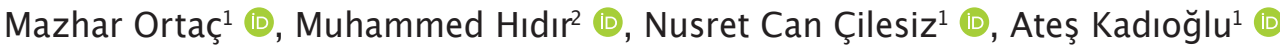

Cite this article as: Ortaç M, Hıdır M, Çilesiz NC, Kadığlu A. Efficacy of follitropin-alpha versus human menopausal gonadotropin for male patients with congenital hypogonadotropic hypogonadism. Turk J Urol 2020; 46(1): 13-7.

ORCID IDs of the authors: M.0.0000-0002-4784-5209; M.H.0000-0002-9232-4621; N.C.C. $0000-0003-2115-698 X$ A.K.0000-0002-4239-7638.

${ }^{1}$ Department of Urology, İstanbul University İstanbul School of Medicine, İstanbul, Turkey

${ }^{2}$ Clinic of Urology, Batman State Hospital, Batman, Turkey

Submitted:

07.09.2019

Accepted:

06.11.2019

Available Online Date:

29.11.2019

Corresponding Author:

Mazhar Ortaç

E-mail:

m_ortac@hotmail.com

(C) Copyright 2020 by Turkish

Association of Urology

Available online at

www.turkishjournalofurology.com

\begin{abstract}
Objective: To compare human menopausal gonadotropin (hMG) and recombinant follicle-stimulating hormone ( $\mathrm{rFSH}$ ) with respect to successful spermatogenesis and pregnancy outcomes in patients with congenital hypogonadotropic hypogonadism $(\mathrm{CHH})$.

Material and methods: This retrospective study included a total of 112 male patients with $\mathrm{CHH}$. Of these, 70 were to receive treatment with hMG and 42 with $\mathrm{rFSH}$ following the hCG administration.

Results: The average age at diagnosis was 27.9 (range, 15-51) years. The baseline luteinizing hormone ( $\mathrm{LH})$, follicle-stimulating hormone (FSH), and testosterone levels were $0.53 \pm 0.77 \mathrm{IU} / \mathrm{L}, 0.63 \pm 0.61 \mathrm{IU} / \mathrm{L}$, and $1.10 \pm 1.90 \mathrm{ng} / \mathrm{dL}$, respectively. Following the combined hormonal treatment, 85.7\% (96/112) of patients had sperm detected in ejaculate samples. In the hMG group, the mean baseline of a testicular size was slightly lower than in the rFSH group $(5.0 \pm 3.5 \mathrm{~mL}$ and $5.3 \pm 3.9 \mathrm{~mL})$, whereas these differences were not statistically significant $(\mathrm{p}=0.364)$. The mean baseline age, level of FSH, LH, and testosterone also showed no significant difference between the two treatment options. The rate of successful spermatogenesis was similar (85.7\%) in both groups, while the pregnancy rates of patients who underwent hMG and $\mathrm{rFSH}$ treatments were $38.6 \%$ $(n=27)$ and $51.2 \%(n=21)$; however, these differences were not statistically significant $(p=0.314)$. No patients developed severe effects during the treatment period.
\end{abstract}

Conclusion: Successful spermatogenesis and pregnancy rates with hMG and rFSH are similar.

Keywords: Follitropin-alpha; gonadotropin; male hypogonadotropic hypogonadism; spermatogenesis.

\section{Introduction}

A normal development of fertility capacity requires the pulsatile secretion of gonadotropinreleasing hormone $(\mathrm{GnRH})$ from the hypothalamus, which also stimulates the synthesis of gonadotropins [luteinizing hormone ( $\mathrm{LH})$, follicle-stimulating hormone (FSH)] from the anterior pituitary gland. ${ }^{[1]}$ The failure of gonadotropin secretion results in clinical hypogonadotropic hypogonadism (HH). Regardless of its etiology, $\mathrm{HH}$ is one of the few causes of male infertility treatable with hormone replacement therapy. In fact, there have been several clinical studies in which patients receiving gonadotropin therapy develop secondary sexual characteristics, spermatogenesis, and testicular enlargement. ${ }^{[2,3]}$
The administration of LH combined with FSH has been successfully used in treating patients with HH. A previous commercial preparation of FSH for therapeutic use was derived from the urine of postmenopausal women that was labeled human menopausal gonadotropin (hMG).$^{[4]}$ The hMG containing both $\mathrm{LH}$ and FSH activity has been widely used since the 1960s. Over the last several decades, a recombinant form of human FSH (rFSH) has been used, instead of urinary human menopausal gonadotropin, after the development of recombinant DNA technology. In 1995, follitropin alfa was licensed for clinical use in the European Union as the first rFSH because it demonstrated pure FSH activity in contrast to hMG. Despite the LH activity, hMG does not maintain 
the Leydig cell function, as well as successful spermatogenesis; therefore, its use in combination with hCG is required to achieve spermatogenesis in patients with $\mathrm{HH}^{[5]}$

According to the literature, several clinical studies have investigated the effect of gonadotropins on spermatogenesis in men with HH. ${ }^{[6]}$ However, to the best of our knowledge, there are no comparative studies considering the efficacy of the urinederived or recombinant FSH on the treatment of male HH. In female patients, data show no advantage of recombinant FSH over urinary gonadotropins (urinary FSH and hMG combined) in terms of pregnancy rate per started cycle in female patients who underwent ovarian hyperstimulation. ${ }^{[7]}$ However, urinary gonadotropins are more cost-effective than $\mathrm{rFSH}$ per ongoing pregnancy, but $\mathrm{rFSH}$ is associated with lower immunologic reactions in contrast to $\mathrm{hMG}{ }^{[8,9]}$

In clinical practice, the traditional treatment protocol includes the administration of hCG (1000-1500 IU) and FSH (75-150 IU), both 2-3 times per week. ${ }^{[10]}$ There is no ideal pharmaceutical dosage of $\mathrm{HH}$ treatment in the literature. Studies on this topic lack the necessary patient population due to the low incidence of the disease and heterogeneity of the treatment options; therefore, a gold standard medical treatment remains to be determined. The aim of this study was to compare the efficacy of rFSH versus hMG for successful spermatogenesis and spontaneous pregnancy in 112 patients diagnosed with $\mathrm{HH}$.

\section{Material and methods}

A total of 112 azoospermic patients diagnosed with congenital hypogonadotropic hypogonadism $(\mathrm{CHH})$ were included in this retrospective study. The diagnosis of $\mathrm{CHH}$ included physical examination, low testosterone levels with low/inappropriately normal FSH/LH values, normal prolactin levels, and azoospermia. Anatomical hypophysis lesions shown by magnetic resonance imaging were an exclusion criterion. Patients did not undergo genetic testing for diagnosed Kallmann syndrome or normosmic idiopathic hypogonadotropic hypogonadism. Clinical findings, including testicular volumes, a history of undescended testis, and medical and family histories, were recorded on enrollment. Average values of both testicular volumes were recorded via a Prader orchidometer (ASSI, Westbury, NY, USA). Semen samples were obtained via masturbation with visual erotic stimulation at the hospital. All semen samples were analyzed in the andrology laboratory according to the criteria of the World Health Organization laboratory manual for the examination and processing of human semen from 1999 (before 2010) and 2010 (after 2010). ${ }^{[11]}$ Patients diagnosed with $\mathrm{CHH}$ were administered LH and FSH analogs as hormone replacement therapy.
The study was conducted in accordance with the principles of the Declaration of Helsinki and approved by the Institutional Review Board (IRB) of Istanbul University, Istanbul Medical Faculty, and informed consent was waived for this retrospective study.

\section{Treatment protocol}

Urine-derived gonadotrophin therapy, which had been in use until 2010, was changed to a recombinant form. Intramuscular hCG treatment started with a dose of $1500 \mathrm{IU}$ (twice per week) and adjusted according to testosterone levels and testicular development over 6 months. If the patient remained azoospermic at the end of 6 months, the FSH treatment (75-150 IU, twice per week) was added to the regime. Target FSH levels of 4-6 IU were achieved by the FSH dose adjustments. The follow-up protocol included the quarterly assessment of semen and FSH and testosterone levels. The recombinant forms of FSH were Gonal F (Merck-Serono, Geneva, Switzerland), and the urine forms of FSH were Menogon (Ferring, GmbH, Kiel, Germany).

\section{Statistical analyzes}

The Statistical Package of Social Sciences for Windows Version 20 (IBM Corp., Armonk, NY, USA) was used for statistical analyzes. We divided patients into two groups based on the treatment options. Categorical variables were presented as numbers and percentages and compared using the chi-squared test. Continuous variables were presented as means and standard deviations and compared using the independent sample t-test. Dependent variables were examined by paired samples t-test. Statistical significance was considered when the two-tailed value of $\mathrm{p}<0.05$.

\section{Results}

A total of 112 azoospermic $\mathrm{CHH}$ patients were treated with hMG (hMG group, $n=70$ patients) or $\mathrm{rFSH}$ (rFSH group, $\mathrm{n}=42$ patients) following the hCG administration. The average age at diagnosis of these patients was 27.9 (range, 15-51) years. The baseline LH, FSH, and testosterone levels were $0.53 \pm 0.77$ $\mathrm{IU} / \mathrm{L}, 0.63 \pm 0.61 \mathrm{IU} / \mathrm{L}$, and $1.10 \pm 1.90 \mathrm{ng} / \mathrm{dL}$, respectively. Fifteen patients (13.4\%) had a history of cryptorchidism. The mean baseline testicular size was $5.16 \pm 2.43 \mathrm{~mL}$ lower than the normal expected testicular size. Following the combined hormonal treatment, 85.7\% (96/112) of patients had sperm detected in ejaculate samples.

Patients were divided into two groups according to the treatment options (hMG or $\mathrm{rFSH}$ ); the mean baseline of testicular size of patients treated with $\mathrm{hMG}$ and $\mathrm{rFSH}$ was $5.0 \pm 3.5 \mathrm{~mL}$ and $5.3 \pm 3.9 \mathrm{~mL}$, respectively. However, these differences were not statistically significant $(\mathrm{p}=0.364)$. For the mean baseline age, 


\section{Table 1. Comparison of patients treated with hMG and rFSH}



and the level of FSH, LH, and testosterone, there was no significant difference between the two treatment options. Furthermore, the number of patients with undescended testes showed no significant difference $(\mathrm{p}=0.780)$.

The rate of successful spermatogenesis in both groups was simi$\operatorname{lar}(85.7 \%)$, while the pregnancy rates of women whose partners underwent hMG and rFSH treatments were $38.6 \%(n=27)$ and $51.2(n=21)$; however, these differences were not statistically significant $(\mathrm{p}=0.314)$ (Table 1$)$.

When patients who had a history of undescended testes were evaluated, the rate of successful spermatogenesis was $73.3 \%$ $(11 / 15)$. While 5 of these patients used recombinant FSH, the reminder used urinary FSH. The rate of successful spermatogenesis was $70 \%(7 / 10)$ in the urine FSH group and $80 \%(4 / 5)$ in the recombinant group. This difference was not statistically significant ( $\mathrm{p}>0.05)$. According to the safety evaluation, no patients developed severe effects during the treatment period.

\section{Discussion}

The LH+FSH combination is an effective therapy for restoring spermatogenesis in male patients with gonadotrophin deficiency. In this retrospective study, we investigated the efficacy and safety of rFSH vs hMG gonadotropin therapy for the restoration of spermatogenesis and pregnancy outcome in 112 patients with $\mathrm{HH}$. In addition, the potential effect of baseline factors on treatment outcomes was also investigated. Approximately, 85.7\% $(96 / 112)$ of the patients had detectable sperm in ejaculates during treatment. Dwyer et al. ${ }^{[12]}$ and the European Metrodin HP
Study Group reported that the FSH/hCG treatment induced spermatogenesis in $84 \%$ and $89.3 \%$ of patients with $\mathrm{HH}$, respectively. ${ }^{[13]}$ In addition, Efesoy et al. ${ }^{[14]}$ reported that successful spermatogenesis was provided with combined hCG+recombinant FSH in 15 of $21(71.4 \%)$ patients with HH. In a meta-analysis of 30 studies by Rastrelli et al. ${ }^{[15]}$, the overall success rate of gonadotropin therapy in gonadotrophin-deficient male patients was $75 \%$ (range, $69 \%-81 \%$ ).

Cryptorchidism is a common anomaly of male genitalia, estimated to affect $2 \%-4 \%$ of full-term male infants, and is a significant prognostic factor for fertility in both the general population and $\mathrm{HH}$ patients. A history of undescended testes almost always negatively affects the restoration of sperm in patients with $\mathrm{HH}^{\left[{ }^{[16]}\right.}$ In this study, we found that $\mathrm{HH}$ patients with cryptorchidism had a lower probability of achieving spermatogenesis. However, both urinary and recombinant FSH showed a similar outcome in terms of spermatogenesis.

Recombinant FSH and $\mathrm{hMG}$ are the gonadotrophin products used most widely to induce spermatogenesis in male patients with $\mathrm{HH}$. Before the availability of recombinant $\mathrm{FSH}$ on the market and highly purified FSH and hMG were the sources of exogenous FSH activity. While hMG contains both the LH and FSH activity, the amount of its LH activity is low. ${ }^{[17]}$ Despite the recombinant production of simple proteins, FSH needs glycosylation for the highest biological activity during recombinant technology. Recombinant human FSH was produced by genetically engineered mammalian cells (Chinese hamster ovary). On the other hand, urine collection is not required for the production of $\mathrm{rFSH} .{ }^{[18]}$ 
There have been numerous studies comparing $\mathrm{rFSH}$ and urinary gonadotrophin administered in female patients who underwent assisted reproduction treatment; ${ }^{[19]}$ however, to the best of our knowledge, this is the first clinical study carried out to compare rFSH and hMG in male patients with HH. In the present study, comparing $\mathrm{rFSH}$ versus hMG overall did not result in any difference in successful spermatogenesis, pregnancy outcome, and any of the other outcomes, including time to first sperm appearance in the ejaculate. In female patients who underwent IVF/ ICS, the Cochrane database included 42 trials in a total of 9606 couples. They showed that there were no differences between rFSH with urinary gonadotrophins in terms of the live birth rate or any of the other outcomes. ${ }^{[7]}$

A meta-analysis of 35 studies by Rastrelli et al. ${ }^{[15]}$ evaluated 20 articles measuring the efficacy of urinary FSH, 10 articles measuring recombinant $\mathrm{FSH}$, and 5 articles measuring highly purified FSH for patients with $\mathrm{CHH}$. They showed that the rate of sperm appearance in the ejaculate was $77 \%, 81 \%$, and $84 \%$ for patients who underwent urinary, highly purified, and recombinant FSH, respectively. These differences were not statistically significant. They also showed that there were no differences between the two FSH options in terms of sperm concentration and pregnancy outcomes. In addition, Rastrelli et al. ${ }^{[15]}$ mentioned that urinary-derived preparations have an advantage according to the cost analysis.

The cost-effectiveness analysis of both treatments may be an important factor in daily clinical preference. The main limitations of the study are retrospective in nature and lack a comparative cost-effectiveness calculation.

In conclusion, the current medical approach for male patients with $\mathrm{HH}$ provides spermatogenesis successful enough for the achievement of pregnancy. The patients can achieve successful spermatogenesis via either $\mathrm{rFSH}$ or $\mathrm{hMG}$, and there is no difference in outcomes between the two treatment regimes. However, using urinary FSH may be a more cost-effective treatment option than the recombinant form. Randomized controlled trials with greater patient numbers are required for future studies.

Ethics Committee Approval: Authors declared that the research was conducted according to the principles of the World Medical Association Declaration of Helsinki "Ethical Principles for Medical Research Involving Human Subjects”, (amended in October 2013).

Informed Consent: N/A.

Peer-review: Externally peer-reviewed.

Author Contributions: Concept - M.O., M.H.; Design - M.O., M.H., N.C.Ç.; Supervision - A.K.; Resources - M.H., N.C.Ç.; Materials -
M.O., M.H.; Data Collection and/or Processing - M.O., M.H.; Analysis and/or Interpretation - M.O., N.C.Ç.; Literature Search - M.O.; Writing Manuscript - M.O., A.K.; Critical Review - A.K.

Conflict of Interest: The authors have no conflicts of interest to declare.

Financial Disclosure: The authors declared that this study has received no financial support.

\section{References}

1. O'Shaughnessy PJ: Hormonal control of germ cell development and spermatogenesis. Semin Cell Dev Biol 2014;29:55-65. [CrossRef]

2. Boehm U, Bouloux PM, Dattani MT, de Roux N, Dode C, Dunkel L, et al. Expert consensus document: European Consensus Statement on congenital hypogonadotropic hypogonadism--pathogenesis, diagnosis and treatment. Nat Rev Endocrinol 2015;11:54764. [CrossRef]

3. Attia AM, Abou-Setta AM, Al-Inany HG: Gonadotrophins for idiopathic male factor subfertility. Cochrane Database Syst Rev 2013:CD005071. [CrossRef]

4. Corona G, Rastrelli G, Maggi M. The pharmacotherapy of male hypogonadism besides androgens. Expert Opin Pharmacother 2015;16:369-87. [CrossRef]

5. Lunenfeld B, Bilger W, Longobardi S, Alam V, D'Hooghe T, Sunkara SK. The Development of Gonadotropins for Clinical Use in the Treatment of Infertility. Front Endocrinol (Lausanne) 2019;10:429. [CrossRef]

6. Pitteloud N, Dwyer A. Hormonal control of spermatogenesis in men: therapeutic aspects in hypogonadotropic hypogonadism. Ann Endocrinol (Paris) 2014;75:98-100. [CrossRef]

7. van Wely M, Kwan I, Burt AL, Thomas J, Vail A, Van der Veen $\mathrm{F}$, et al. Recombinant versus urinary gonadotrophin for ovarian stimulation in assisted reproductive technology cycles. Cochrane Database Syst Rev 2011:CD005354. [CrossRef]

8. Phipps WR, Holden D, Sheehan RK. Use of recombinant human follicle-stimulating hormone for in vitro fertilization-embryo transfer after severe systemic immunoglobulin E-mediated reaction to urofollitropin. Fertil Steril 1996;66:148-50. [CrossRef]

9. Goldfarb JM, Desai N. Follitropin-alpha versus human menopausal gonadotropin in an in vitro fertilization program. Fertil Steril 2003;80:1094-9. [CrossRef]

10. Silveira LF, Latronico AC. Approach to the patient with hypogonadotropic hypogonadism. J Clin Endocrinol Metab 2013;98:17818. [CrossRef]

11. World Health Organization. WHO laboratory manual for the examination and processing of human semen, 5th edn. Geneva: World Health Organization; 2010.

12. Dwyer AA, Raivio T, Pitteloud N. Gonadotrophin replacement for induction of fertility in hypogonadal men. Best Pract Res Clin Endocrinol Metab 2015;29:91-103. [CrossRef]

13. Efficacy and safety of highly purified urinary follicle-stimulating hormone with human chorionic gonadotropin for treating men with isolated hypogonadotropic hypogonadism. European Metrodin HP Study Group. Fertil Steril 1998;70:256-62 . [CrossRef] 
14. Efesoy O, Cayan S, Akbay E. The efficacy of recombinant human follicle-stimulating hormone in the treatment of various types of male-factor infertility at a single university hospital. J Androl 2009;30:679-84. [CrossRef]

15. Rastrelli G, Corona G, Mannucci E, Maggi M. Factors affecting spermatogenesis upon gonadotropin-replacement therapy: a metaanalytic study. Andrology 2014;2:794-808. [CrossRef]

16. Ortac M,Hidir M, Salabas E, Boyuk A, Bese C, Pazir Y, et al.Evaluation of gonadotropin-replacement therapy in male patients with hypogonadotropic hypogonadism. Asian J Androl 2019;21:623-7. [CrossRef]
17. Loumaye E, Campbell R, Salat-Baroux J. Human follicle-stimulating hormone produced by recombinant DNA technology: a review for clinicians. Hum Reprod Update 1995;1:188-99. [CrossRef]

18. Hugues JN. Recombinant human follicle-stimulating hormone: a scientific step to clinical improvement. Reprod Biomed Online 2001;2:54-64. [CrossRef]

19. Al-Inany HG, Abou-Setta AM, Aboulghar MA, Mansour RT, Serour GI. Efficacy and safety of human menopausal gonadotrophins versus recombinant FSH: a meta-analysis. Reprod Biomed Online 2008;16:81-8. [CrossRef] 